\title{
Intrabullous blood injection for lung volume reduction
}

\author{
S Kanoh, H Kobayashi, K Motoyoshi
}

Division of Pulmonary Medicine, Department of Medicine, National Defense Medical College, Tokorozawa, Saitama, Japan

Correspondence to: Dr S Kanoh, Division of Pulmonary Medicine, Department of Medicine, National Defense Medical College, 3-2 Namiki, Tokorozawa, Saitama 359-8513, Japan; kanoh@ndmc.ac.jp

Received 26 July 2007 Accepted 24 October 2007

\section{ABSTRACT}

Bronchoscopic treatment for emphysematous lung diseases has attracted clinical attention, and several different approaches are being investigated. We present a case of emphysematous bullae that was effectively treated with a newly developed bronchoscopic intervention, autologous blood injection. A 59-year-old man was referred to our institution with exertional dyspnoea. Chest CT showed emphysema and bullae with a diameter of $12 \mathrm{~cm}$ in the right upper lobe. Bronchoscopic treatment was introduced as an alternative to surgery. Autologous blood and fibrinogen solution were infused into bullae via the transbronchial catheter, under fluoroscopic guidance. Post-treatment CT showed marked contraction of bullae to a diameter of $3 \mathrm{~cm}$, corresponding to a volume reduction of $800 \mathrm{ml}$ on body plethysmography. A significant reduction in dyspnoea was also noted. This therapeutic approach is less invasive and may represent a good option for reducing lung volume.

In patients with emphysematous bullae, surgical bullectomy has been the treatment of choice, depending on size, location and intensity of symptoms. ${ }^{1}$ Indeed, bullectomy provides significant improvements in dyspnoea, gas exchange and pulmonary function. ${ }^{2}$ However, the surgical procedure is not without risks of both complications and mortality. ${ }^{1}$ Less invasive and safer alternative treatments would thus be preferable. A recent report has shown that bullae can be successfully treated by bronchoscopic placement of one way valves for endobronchial blockage. ${ }^{3}$ Bronchoscopic lung volume reduction for emphysematous lung diseases is under development, and several different approaches are currently being investigated..$^{3-5}$ This report represents the first description of emphysematous bullae treated by transbronchial infusion of autologous blood.

\section{CASE REPORT}

A 59-year-old man was referred to our institution with increasing dyspnoea on exertion and a diagnosis of chronic obstructive pulmonary disease and emphysematous bullae. The patient was treated using inhalation of salmeterol and budesonide. The patient had an 80 pack-year smoking history, but had stopped smoking 4 years earlier. Physical examination was unremarkable except for diminished breath sounds. Arterial blood gas analysis showed: $\mathrm{pH}$ 7.411, $\mathrm{PaCO}_{2} 41.5 \mathrm{~mm} \mathrm{Hg}$ and $\mathrm{PaO}_{2} 71.1 \mathrm{~mm} \mathrm{Hg}$. Pulmonary function tests demonstrated obstructive ventilatory impairment with: peak expiratory flow (PEF) 6.14 l/s (74\% predicted); forced expiratory volume in $1 \mathrm{~s}\left(\mathrm{FEV}_{1}\right)$ 1.941 (69\% predicted); and $\mathrm{FEV}_{1} /$ forced vital capacity (FVC) ratio 59\%. Transfer factor for carbon monoxide (Tlco) was $68 \%$ predicted.
Lung volumes measured by body plethysmography showed: total lung capacity (TLC) 6.59 l (116\% predicted); intrathoracic gas volume (TGV) 4.031 (123\% predicted); and residual volume (RV) 2.851 (150\% predicted). Chest radiography showed bullae with a diameter of $12 \mathrm{~cm}$ in the right upper lung field. Chest CT confirmed the presence of several large bullae in the right upper lobe and multiple small low attenuation areas.

The patient refused surgical bullectomy and therefore bronchoscopic treatment was proposed for relief from dyspnoea. Informed consent was obtained in writing after providing a sufficient explanation, and the Institutional Review Board of Ethics approved the treatment protocol.

Transbronchial treatment using a flexible bronchoscope was performed under topical anaesthesia. The bronchoscope was introduced into the right upper lobe bronchus. Next, a catheter (PW1L-1; Olympus, Tokyo, Japan) was inserted through the working channel of the bronchoscopy into the right upper apical segmental bronchus and advanced under fluoroscopic guidance until the catheter was positioned inside the bulla. During the procedure, a total of $10 \mathrm{ml}$ of autologous blood were obtained through an intravenous catheter in the upper limb and subsequently infused into the bullae via the intrabronchial catheter. Successively, $3 \mathrm{ml}$ of fibrinogen and thrombin solution (CSL Behring, King of Prussia, Pennsylvania, USA) were infused. The entire procedure was completed in 5 min without incident. Although transient fever and cough were noted on the same day, the condition of the patient remained stable.

After the procedure, a significant reduction in dyspnoea was noted. Spirometry showed improvements with: PEF $7.04 \mathrm{l} / \mathrm{s}$ (85\% predicted); $\mathrm{FEV}_{1}$ 2.181 (78\% predicted); and $\mathrm{FEV}_{1} / \mathrm{FVC}$ ratio $63 \%$. Tlco had risen to $74 \%$ predicted whereas plethysmography revealed reductions in lung volumes, as follows: TLC 5.761 ; TGV 3.241 ; and RV 2.081. This result indicates that lung volume decreased by $800 \mathrm{ml}$. These improvements were also supported radiologically, and post-treatment coronal reconstructed CT showed marked contraction of bullae with a diameter of $3 \mathrm{~cm}$ (fig 1). A bulla was shrunken from approximately $75 \mathrm{~cm}^{2}$ to $6 \mathrm{~cm}^{2}$. Twelve months after the bronchoscopic treatment, the patient's symptom remained in remission and free from exertional dyspnoea.

\section{DISCUSSION}

Enlarged bullae compress the surrounding lung and limit respiratory function. Removal of bullae can thus result in improved lung function and thereby quality of life. ${ }^{1-3}$ Endobronchial valve replacement is the treatment of choice but is not available everywhere. ${ }^{3}$ Ingenito and colleagues ${ }^{5}$ developed 

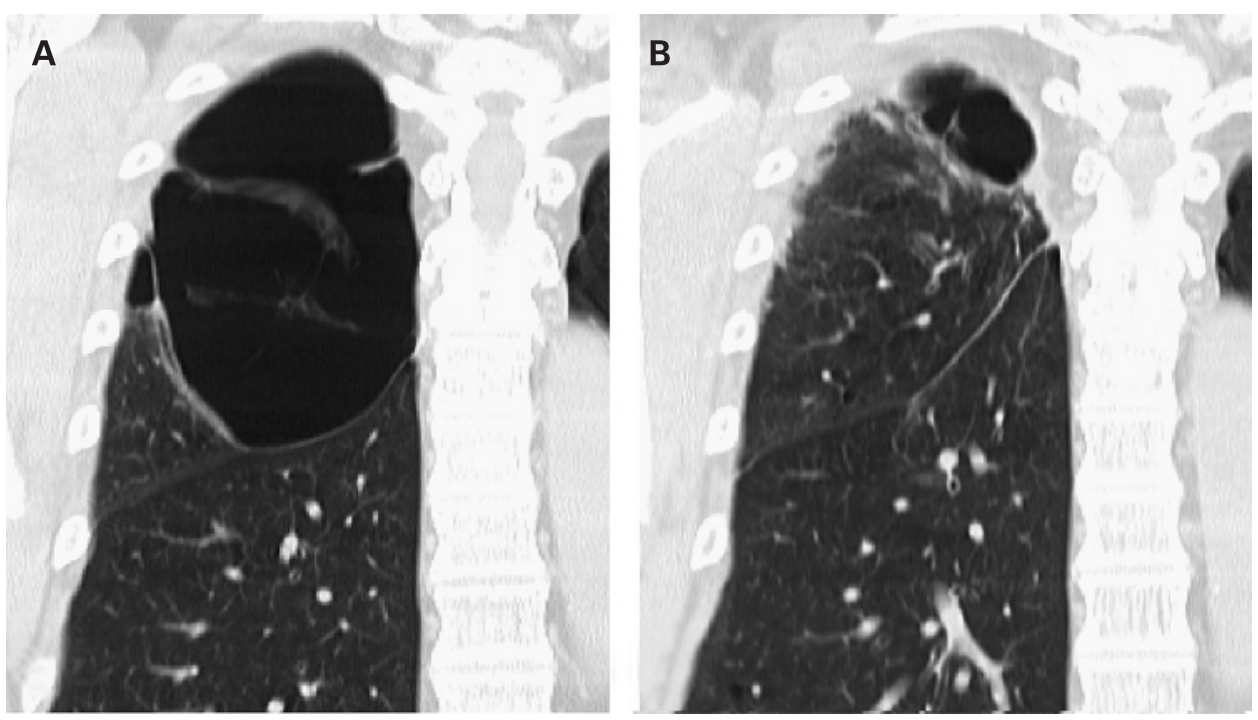

Figure 1 Coronal reformatted CT image taken before $(A)$ and after $(B)$ treatment with autologous blood injection. After bronchoscopic treatment, a marked contraction of bulla was demonstrated, which shrunk from 12 to $3 \mathrm{~cm}$ in diameter.

bronchoscopic lung volume reduction using biologically active reagents delivered through a flexible bronchoscope to promote scar formation in experimental emphysema in sheep. In patients with advanced emphysema, a clinical trial based on this system is currently in progress. ${ }^{6}$ We applied a modified procedure using blood infusion to emphysematous bullae, under Institutional Review Board approval, and demonstrated successful contraction of bullae.

Firstly, we used autologous blood as an impetus. Although no previous reports have described lung volume reduction by injecting blood, blood has potential bioadhesive properties. ${ }^{7}$ For example, autologous "blood patch" has been used for the treatment of pneumothorax. ${ }^{8}$ Hence clot formation may lead to collapse of emphysematous alveoli by plugging and producing scarring. Secondly, fibrinogen and thrombin would enhance this effect and promote fibroblast attachment and collagen synthesis, followed by contracted organisation in the alveolar space..$^{4-6}$ Biological glue generated by the interaction between fibrinogen and thrombin is widely used in clinical practice. In therapeutic bronchoscopy, fibrin glue is administered to stop the air leak from bronchopleural fistula. ${ }^{9}$ Also, thrombin infusion into a bronchus is an established technique in the treatment of haemoptysis. ${ }^{10}$ These historical evidences are the rational bases of current clinical attempt. As a result, we achieved significant reduction of emphysematous bullae, leading to marked functional and symptomatic relief. Radiological differences in chest CT imaging between pre- and post-treatment were notable and sufficient to confirm the effectiveness of this reformative therapy. Furthermore, this method is less invasive, easy and inexpensive.
In conclusion, bronchoscopic treatment of bullae by infusion of autologous blood can offer an effective therapeutic option and warrants further investigation in large cases.

Competing interests: None.

Ethics approval: The Institutional Review Board of Ethics approved the treatment protocol.

\section{REFERENCES}

1. Meyers BF, Patterson GA. Bullectomy, lung volume reduction surgery, and transplantation for patients with chronic obstructive pulmonary disease. Thorax 2003; 58:634-8.

2. Palla A, Desideri M, Rossi G, et al. Elective surgery for giant emphysema: a 5-year clinical and functional follow up. Chest 2005;128:2043-50.

3. Nappen M, Tellings J-C, Dekeukeleire T, et al. Successful treatment of a giant emphysematous bulla by bronchoscopic placement of endobronchial valves. Chest 2006;130:1563-5.

4. Ingenito EP, Tsai LW. Bronchoscopic lung volume reduction. In: Simoff MJ, Sterman $\mathrm{DH}$, Ernst A, eds. Thoracic endoscopy: advances in interventional pulmonology. Malden, MA: Blackwell Publishing, 2006:183-96.

5. Ingenito EP, Berger RL, Henderson AC, et al. Bronchoscopic lung volume reduction using tissue engineering principles. Am J Respir Crit Care Med 2003;167:771-8.

6. Reilly J, Washko G, Pinto-Plata V, et al. Biological lung volume reduction: A new bronchoscopic therapy for advanced emphysema. Chest 2007;131:1108-13.

7. Anitua E, Andia I, Ardanza B, et al. Autologous platelets as a source of proteins for healing and tissue regeneration. Thromb Haemost 2004;91:4-15.

8. Dumire R, Crabbe MM, Mappin FG, et al. Autologous "blood patch" pleurodesis for persistent pulmonary air leak. Chest 1992;101:64-6.

9. Matar AF, Hill JG, Duncan W, et al. Use of biological glue to control pulmonary air leaks. Thorax 1990;45:670-4.

10. Tsukamoto T, Sasaki H, Nakamura H. Treatment of hemoptysis patients by thrombin and fibrinogen-thrombin infusion therapy using a fiberoptic bronchoscope. Chest 1989;96:437-76 